\title{
Las Hidroeléctricas: efectos en los ecosistemas y en la salud ambiental
}

\section{Hydroelectric Dams: effects on ecosystems and environmental health}

\author{
Edgar-Ricardo Oviedo-Ocaña ${ }^{1}$
}

Forma de citar: Oviedo-Ocaña, ER. Las Hidroeléctricas: efectos en los ecosistemas y en la salud ambiental. Rev Univ Ind Santander Salud. 50(3): 191-192. doi: http://dx.doi.org/10.18273/revsal.v50n3-2018003 @) (1)

La creciente demanda de energía asociada al desarrollo económico y el crecimiento demográfico, ha llevado a estrategias que priorizan la expansión de la producción de energía eléctrica. En Colombia se estima un crecimiento promedio anual de la demanda de aproximadamente $2.9 \%$. La construcción de hidroeléctricas se ha constituido en uno de los principales mecanismos de producción energética, representando alrededor del $70 \%$ de la generación total en el país. Comúnmente, la producción de energía eléctrica mediante hidroeléctricas se ha denominado como energía limpia y renovable ${ }^{1}$. No obstante, los impactos sociales y ambientales de la construcción de presas, así como los efectos en la salud de la población han sido reconocidos desde hace mucho tiempo ${ }^{2,3}$.

La retención de agua en los reservorios modifica el régimen hidrológico e hidráulico de las corrientes de agua, de lotico (aguas fluviales) a lentico (aguas superficiales de muy bajo flujo como los lagos), afectando los procesos de escorrentía, de transporte de sedimentos y cambiando la geomorfología de los ríos antes y después de las estructuras. La descomposición de la materia orgánica en los embalses puede promover la generación de gases de efecto invernadero (GEI) como el metano (se estima que las represas son las responsables de $1.3 \%$ de las emisiones globales de GEI). Así mismo, la concentración de macronutrientes en aguas lenticas, podría incrementar la producción de fitoplancton el cual reduce la concentración de oxígeno disuelto y la calidad del agua e incrementa la biomasa (fenómeno conocido como eutroficación). Adicionalmente, la presión del agua sobre el suelo podría alterar su estabilidad, generando deslizamientos o sismicidad inducida ${ }^{2,4}$.

Estos cambios en las condiciones hidráulicas del agua afectan significativamente la diversidad biótica en ecosistemas ribereños. Generalmente, se produce la disminución de especies nativas y se promueve la diseminación anómala de especies exóticas más adaptadas a las condiciones lenticas. Además, el represamiento de los ríos dificulta la libre circulación de especies migratorias causando la fragmentación del hábitat. La interrupción de la conectividad también reduce los sitios de desove, reproducción y sus habitas, afectando la población diversa de fauna ${ }^{2}$. La disminución en la concentración de oxígeno disuelto causa la mortandad de peces y otras especies acuáticas. De igual manera, las inundaciones por el llenado del reservorio, generan una pérdida de flora ribereña, migración de especies de aves, reptiles y mamíferos, que pierden su hábitat y los espacios para la alimentación.

La transformación de los ecosistemas genera afectaciones en la producción y modo de vida de las poblaciones humanas que deben ser desplazadas, no solo del espacio que será inundado sino de áreas aledañas. Las actividades de sustento como la pesca deben ser modificadas debido al impacto que tiene la infraestructura sobre la población de peces. Esto atenta contra la seguridad y autonomía alimentaria de las poblaciones, cuyos medios de sustento dependen cada vez más del mercado externo ${ }^{5}$. Así mismo, debido al desplazamiento de la población, las nuevas áreas requeridas para el desarrollo de actividades agrícolas y pecuarias implican transformaciones de la cobertura del suelo que conlleva una reducción significativa de los bosques primarios ${ }^{6}$.

Los impactos de los embalses en el ecosistema y en los medios de sustento, afectan la salud de la población. Acorde con Lerer y Scudder ${ }^{3}$, entre los potenciales impactos, se tienen: i) Enfermedades asociadas con el agua debido a la pérdida de fuentes hídricas (río o manantiales) las cuales pueden destruirse o secarse, o asociados a las

1. Director del Grupo de Investigación en Recursos Hídricos y Saneamiento Ambiental (GPH). Universidad Industrial de Santander. Bucaramanga. Colombia. 
nuevas condiciones de agua, saneamiento e higiene de poblaciones que han tenido que asentarse en otras áreas; ii) Afectación de la seguridad alimentaria que podría influenciar negativamente la nutrición, especialmente de niños y otras personas vulnerables; iii) Enfermedades de transmisión sexual asociadas al asentamiento temporal de personas durante la etapa de construcción de la infraestructura; iv) Afectación psicológica asociada con la alta actividad sísmica generada por el represamiento de grandes volúmenes de agua, el riesgo de inundaciones en áreas ribereñas o debidos a la pérdida del paisaje y patrimonio natural que quedan sumergidos o perturbados por el embalse.

Una situación adicional asociada a este tipo de proyectos está relacionada con la estimación de la población afectada. En ocasiones, no se tiene en consideración que los impactos de las represas van más allá del área directamente inundada por el embalse ${ }^{1}$. Generalmente, se incluye a la población que debe ser desplazada del área de inundación sin considerar a personas que habitan el área de influencia las cuales se encuentran en condiciones de vulnerabilidad debido a la operación de las hidroeléctricas o a aquellas a las que se les ha limitado el derecho a la autogestión del agua y a coexistir con las dinámicas naturales del río 5 .

De otro lado, aunque las decisiones sobre la construcción de las represas tienen dimensiones técnicas que incluyen las evaluaciones de impacto social y ambiental de estas megaestructuras, experiencias documentadas por Fearnside ${ }^{1}$ muestran que existen presiones por actores con poderes financieros y políticos (incluidos los transnacionales) para la instalación de estos sistemas, que generan condiciones asimétricas en las negociaciones y decisiones. En algunos contextos, la toma de decisiones no favorece la participación real de las personas que serán directamente desplazadas de las áreas de influencia de este tipo de proyectos. Así mismo, las metodologías y prácticas para la estimación de los costos ambientales y sociales asociadas a estos proyectos tienen limitaciones y difícilmente capturan la magnitud de los impactos a diferentes escalas y niveles, y menos compensan de manera justa a los afectados directos de la implementación de este tipo de iniciativas, generalmente los más vulnerables y sin poder.

Es necesaria una discusión sobre la política energética en el país, que considere el acceso a energía para todos, pero considerando energía sostenible, que mejore el rendimiento energético y aumente el uso de otras fuentes renovables. Para tal efecto, es necesario niveles de financiación y compromiso de los países para adoptar tecnologías alternativas en una escala mucho más amplia. Es fundamental promover la investigación y la tecnología relativas a energías que consideren fuentes renovables y eficiencia energética ${ }^{7}$. Experiencias en otros contextos muestran el avance de fuentes alternativas de energía como la eólica, la solar y la generada a partir del tratamiento de residuos sólidos orgánicos y de aguas residuales. De igual manera, es indispensable abordar aspectos sobre modalidades de consumo y producción sostenible, fomentando el uso eficiente de recursos y de la energía, y buscando la reducción de la degradación y la contaminación.

\section{Referencias}

1. Fearnside PM. Environmental and social impacts of hydroelectric dams in Brazilian Amazonia: implications for the aluminum industry. World Development. 2016; 77: 48-65. doi: https://doi.org/10.1016/j.worlddev.2015.08.015.

2. Santos RMB, Fernandes LS, Cortes RMV, Varandas SGP, Jesus JJB, Pacheco FAL. Integrative assessment of river damming impacts on aquatic fauna in a Portuguese reservoir. Sci Total Environ. 2017; 601-602: 1108-1118. doi: 10.1016/j.scitotenv.2017.05.255.

3. Lerer LB, Scudder T. Health impacts of large dams. Environ Impact Assess Rev. 1999;19(2), 113-123. doi: https://doi.org/10.1016/S0195-9255(98)00041-9.

4. Wang QG, Du YH, Su Y, Chen KQ. Environmental impact post-assessment of dam and reservoir projects: a review. Procedia Environ Sci. 2012; 13, 1439-1443.

5. Roa T, Duarte B. Desarrollo hidroeléctrico, despojo y transformación territorial: el caso de Hidrosogamoso, Santander, Colombia. aguas robadas. despojo hídrico y movilización social, Ed: Arroyo A, Boelens R. Instituto de Estudios Peruanos, Lima (Perú); 2013.

6. Jiang X, Lu D, Moran E, Calvi MF, Dutra LV, Li G. Examining impacts of the Belo Monte hydroelectric dam construction on land-cover changes using multitemporal Landsat imagery. Appl Geog. 2018; 97, 35-47. https:// doi.org/10.1016/j.apgeog.2018.05.019.

7. Programa de las Naciones Unidas para el Desarrollo - PNUD. Objetivos de Desarrollo Sostenible. Objetivo 7. Energía Asequible y no contaminante. Objetivo 12. Consumo responsable y Producción; 2015. 